\title{
SPARSITY SEEKING TOTAL GENERALIZED VARIATION FOR UNDERSAMPLED TOMOGRAPHIC RECONSTRUCTION
}

\author{
Daniil Kazantsev ${ }^{1,2}$, Evgueni Ovtchinnikov ${ }^{3}$, Philip J. Withers ${ }^{1,2}$, William R. B. Lionheart ${ }^{4}$, Peter D. \\ Lee $^{1,2}$ \\ ${ }^{1}$ Manchester X-Ray Imaging Facility, The University of Manchester, Manchester, UK \\ ${ }^{2}$ Manchester X-Ray Imaging Facility, Research Complex at Harwell, Didcot, UK \\ ${ }^{3}$ Visualization Group, Research Complex at Harwell, STFC, Oxfordshire, UK \\ ${ }^{4}$ School of Mathematics, Alan Turing Building, The University of Manchester, Manchester, UK
}

\begin{abstract}
Here we present a novel iterative approach for tomographic image reconstruction which improves image quality for undersampled and limited view projection measurements. Recently, the Total Generalized Variation (TGV) penalty has been proposed to establish a desirable balance between smooth and piecewise-constant solutions. Piecewise-smooth reconstructions are particularly important for biomedical applications, where the image surface slowly varies. The TGV penalty convexly combines the first and higher order derivatives, which means that for some regions (e.g. uniform background) it can be more challenging to find a sparser solution due to the weight of the higher order term. Therefore we propose a simple heuristic modification over the Chambolle-Pock reconstruction scheme for TGV which consists of adding the wavelet thresholding step which helps to suppress aliasing artifacts and noise while preserve piecewise-smooth appearance. Preliminary numerical results with two piecewisesmooth phantoms show strong improvement of the proposed method over TGV and TV penalties. The resulting images are smooth with sharp edges and fewer artifacts visible.
\end{abstract}

Index Terms - Iterative reconstruction, regularization, missing wedge, limited data, hard thresholding, wavelets

\section{INTRODUCTION}

Due to clinical restrictions on the acceptable level of radiation dose, the number of projections, or/and the exposure time should be kept to a minimum. This usually results in poor signal-to-noise (SNR) ratio of reconstructed images. In mathematical terms, the undersampled or limited view projection data inversion is an ill-conditioned ill-posed problem

This work has been supported by the Engineering and Physical Sciences Research Council under grants EP/J010456/1 and EP/I02249X/1. We thank Diamond Light Source (DLS) for the access to Diamond-Manchester Branchline. We thank Hua Geng for providing the bioglass sample data and Dr. Thomas Blumensath (Southampton Uni) for useful comments.
[1]. In the case of insufficient noisy measurements, regularized iterative techniques can provide much better quality of reconstructions than analytical methods. Additional regularity on the solution can be imposed through smoothness (e.g. Tikhonov quadratic $\ell_{2}$-norm penalty) or one can assume a sparser solution and use some approximation of $\ell_{1}$-norm, such as the Total Variation (TV) semi-norm [2]. Although TV-based undersampled reconstruction delivers impressive results in removing noise and some artifacts it also produces piecewise-constant images (the so-called "cartoon" effect) even when the original object is smooth. The "cartoon" appearance can be particularly undesirable in emission tomography (ET), where due to limited resolution of the imaging system, reconstructed images are naturally blurred [3]. Since the activity distribution is assumed to be piecewise-smooth, the TV penalty can bias the subsequent clinical interpretation of reconstructed images.

Various attempts have been made to reduce the "cartoon" effect of the recovered images by coupling the first order derivative term (e.g. the TV semi-norm) with higher order derivatives (e.g. the second order as the Laplace operator) [4]. One of the most advanced recent approaches is the Total Generalized Variation (TGV) penalty which convexly combines first and higher order derivatives with different weighting factors [5]. Weighting factors can be found empirically, however we noticed that for some regions (e.g. the uniform background) it is difficult to achieve sparser solutions due to the weight of higher order derivatives.

In this work, to improve the sparsifying properties of the reconstruction with the TGV penalty we add an Iterative Hard Thresholding (IHT) step where small wavelet coefficients (which normally represent noise) are removed [6]. This modification aims at reducing aliasing artifacts from undersampling, artifacts due to limited view and also suppressing noise. The reconstruction algorithm is based on the Chambolle-Pock (CP) primal-dual iterative method [7, 8], which can also be adapted to the convex TGV formalism [4]. 
The IHT operation is applied on every iteration of the CP algorithm using the Fast Wavelet Transform which is computationally efficient. We compare the proposed method Total Generalized Variation Thresholding (TGVT) with the TGV and the classical TV penalty.

\section{METHOD}

The problem of tomographic reconstruction can be formulated as the regularized least squares (LS) minimization:

$$
\begin{aligned}
\hat{\boldsymbol{u}} & =\underset{\boldsymbol{u}}{\operatorname{argmin}} \psi_{\lambda}(\boldsymbol{u}), \\
\psi_{\lambda}(\boldsymbol{u}) & =\frac{\|\mathrm{A} \boldsymbol{u}-\boldsymbol{b}\|_{2}^{2}}{2}+\lambda \operatorname{TGV}_{s}(\boldsymbol{u}),
\end{aligned}
$$

where $\boldsymbol{b} \in \mathbb{R}^{M}$ is a discrete function of the number of the detector bins and the observation angles describing the projection data (sinogram), $\boldsymbol{u} \in \mathbb{R}^{N}$ is a function of spacial variables describing the observed object, $\mathrm{A}: \mathbb{R}^{N} \rightarrow \mathbb{R}^{M}$ is a sparse system projection matrix and $\lambda$ is the regularization parameter. The regularization functional $\mathrm{TGV}_{s}$ is the Total Generalized Variation semi-norm [5], which convexly combines first and higher order derivatives $(s \geq 1)$ using positive weighting parameters $\boldsymbol{\alpha}=\left(\alpha_{1}, \ldots, \alpha_{s-1}\right)$, given as:

$$
\mathrm{TGV}_{s}(\boldsymbol{u})=\sup \left\{-\int_{\Omega} \boldsymbol{u} \operatorname{div}^{s} \boldsymbol{v} \mathrm{d} \boldsymbol{x}\right\}
$$

$$
\boldsymbol{v} \in \mathcal{C}_{c}^{s}\left(\Omega, \operatorname{Sym}^{s}\left(\mathbb{R}^{d}\right)\right),\left\|\operatorname{div}^{l} \boldsymbol{v}\right\|_{\infty} \leq \alpha_{l}, l=(0, \ldots, s-1),
$$

where $\mathcal{C}_{c}^{s}\left(\Omega, \operatorname{Sym}^{s}\left(\mathbb{R}^{d}\right)\right)$ denotes the space of continuously differentiable symmetric $s$ - tensors with compact support in $\Omega \subset \mathbb{R}^{d}$ (in our case $d=2$ ). The $\mathrm{TGV}_{s=2}(\boldsymbol{u})$ can be also written as:

$$
\mathrm{TGV}_{2}(\boldsymbol{u})=\alpha_{1} \int_{\Omega}|\nabla \boldsymbol{u}-\boldsymbol{v}| \mathrm{d} \boldsymbol{x}+\alpha_{0} \int_{\Omega}|\mathcal{E}(\boldsymbol{v})| \mathrm{d} \boldsymbol{x},
$$

where $\mathcal{E}(\boldsymbol{v})=\frac{\nabla \boldsymbol{v}+\nabla \boldsymbol{v}^{\mathrm{T}}}{2}$ is a symmetrized gradient operator. Notably the TGV is equivalent to the TV penalty $\mathrm{TGV}_{1}(\boldsymbol{u})=\mathrm{TV}(\boldsymbol{u})$ when $\boldsymbol{v}=0$ in 3 . The TV penalizes the norm of the gradient and recovery delivers piecewiseconstant solutions. Adding weighting by $\alpha_{0}$ the secondorder term can establish some trade-off between smooth and discontinuous solutions. In this experiment we consider $\mathrm{TGV}_{2}(\boldsymbol{u})(3)$ and $\mathrm{TGV}_{1}(\boldsymbol{u})$ for the TV penalty.

The Chambolle-Pock (CP) primal-dual algorithm [7] is a first-order convex optimization technique based on proximal splitting and can be used to solve (1) [4, 8]. The resulting CP algorithm for the TGV penalty is outlined in Alg. 1.

In Alg. 1, $\operatorname{div}_{\nabla, \mathcal{E}}$ are divergence operators of $\nabla$ and $\mathcal{E}$ operators respectively, and projections onto convex sets $P$ and $Q$ are given as:

$$
\operatorname{proj}_{P}^{\alpha_{1}, \lambda}(\hat{\boldsymbol{p}})=\frac{\hat{\boldsymbol{p}}}{\max \left(\lambda, \frac{\lambda|\hat{\boldsymbol{p}}|}{\alpha_{1}}\right)} ; \operatorname{proj}_{Q}^{\alpha_{0}, \lambda}(\hat{\boldsymbol{q}})=\frac{\hat{\boldsymbol{q}}}{\max \left(\lambda, \frac{\lambda|\hat{\boldsymbol{q}}|}{\alpha_{0}}\right)}
$$

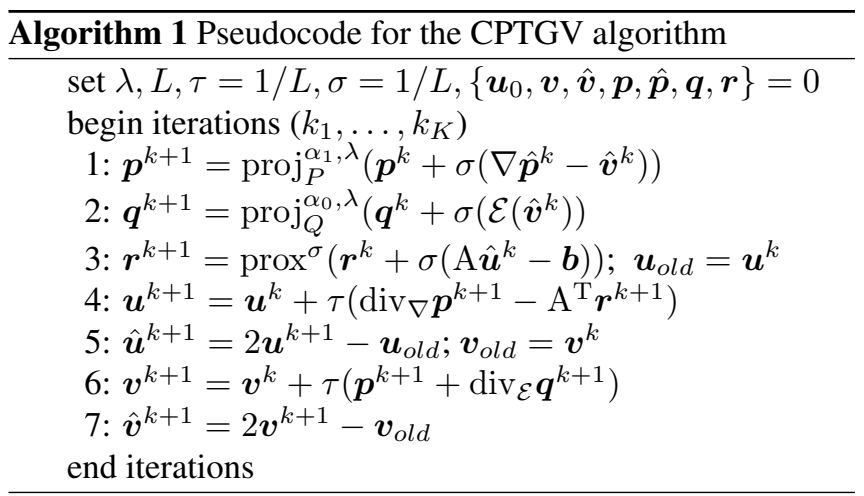

The proximal mapping is given as $\operatorname{prox}^{\sigma}(\hat{\boldsymbol{r}})=\frac{\hat{\boldsymbol{r}}}{1+\sigma}$. To ensure convergence of Alg. 11 we choose $L=16$ [4, 7, 8].

We notice that it is important to choose weighting factors $\alpha_{0,1}$ (3) carefully to establish a good quality of piecewisesmooth reconstruction. Moroever, when the second order term is amplified with a non-optimal choice of $\alpha_{0}$, the reconstruction in uniform areas is dominated by low frequency oscillations. In this case, aliasing artifacts in the background are more prominent in the recovery. To suppress artifacts and further reduce noise we apply the sparsifying wavelet transform on every iteration of the CPTGV algorithm and remove small (unrepresentative) wavelet coefficients.

In [6] it was proposed to use a simple Landweber-like iteration: $\boldsymbol{u}^{k+1}=\Psi\left(\boldsymbol{u}^{k}+\mu \mathrm{A}^{\mathrm{T}}(\mathrm{A} \boldsymbol{u}-b)\right)$ to achieve the solution of the non-convex optimization problem $\operatorname{argmin} \frac{\|\mathrm{A} \boldsymbol{u}-\boldsymbol{b}\|_{2}^{2}}{2}$ s.t. $\|\boldsymbol{u}\|_{0} \leq J$. Here $\Psi(\cdot)$ is a hard thresholding (HT) operator that keeps the largest (in magnitude) $J$ elements of a vector. With an appropriate choice of $\mu$ the IHT algorithm converges to a sub-optimal solution. Here we choose the Dual-Tree Wavelet Transform [9] $\mathcal{H}$ and some threshold $t$ (found empirically in our case): $\Psi_{t}(x)=\mathcal{H}^{-1} \mathrm{HT}(\mathcal{H})$. Next we embedded the IHT operation into step 4 of the CPTGV algorithm which resulted in the CPTGVT method (see Alg. 2).

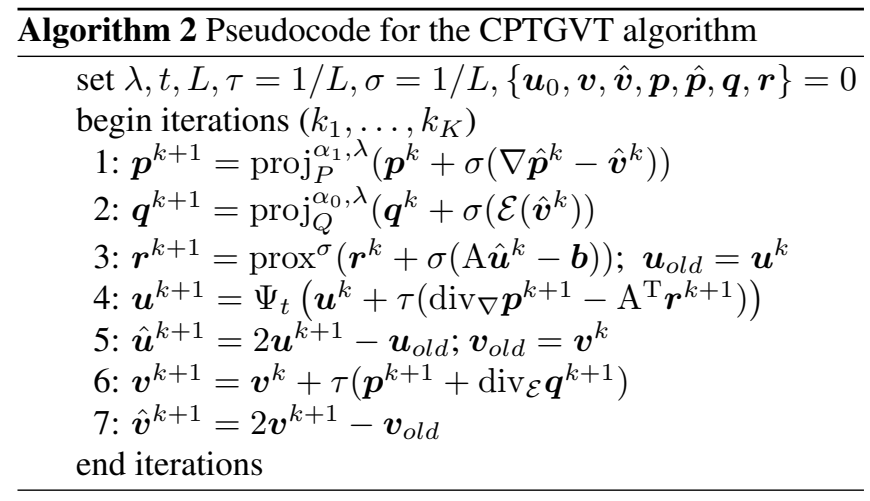

The thresholding step in the line 4 of Alg. 2 comes in accordance with the IHT theory, however the method looses its convexity and convergence is hard to prove. The algorithm is heuristic but reliable in practice provided that $\tau$ is small. 


\section{NUMERICAL EXPERIMENTS}

In this section we compare three $\mathrm{CP}$ reconstruction methods using TV [2, 8], TGV (see Alg. 1) and TGVT (see Alg. 2) penalties. All methods are implemented using the $\mathrm{CP}$ scheme [7] and computer code (Matlab and C-OMP) is provided [10]. To test our techniques, two piecewise-smooth phantoms are used: one is a simple analytical phantom and another phantom is constructed from a real X-ray reconstructed image. All reconstructions are made avoiding the "inverse crime", i.e. different pixel grids have been taken for projection generation and reconstruction, also different projection models (strip and linear) have been employed. Gaussian random noise $(\kappa=$ $5 \%$ of noise to signal ratio) is applied to the simulated projection data. Presented figures and errors are provided after thorough optimization for all parameters $\left(\lambda, \alpha_{0,1}, t\right)$ for each method with respect to the Normalized Root Mean Square Error (NRMSE) $\Delta_{1}$. Notably parameters $\alpha_{0,1}$ for the TGV method were kept the same for the TGVT method as well.

First we test our reconstruction techniques using an analytical phantom (No.1) which consists of two Gaussians, two parabolas and a rectangle (see Fig. 11 (top row)). For op-

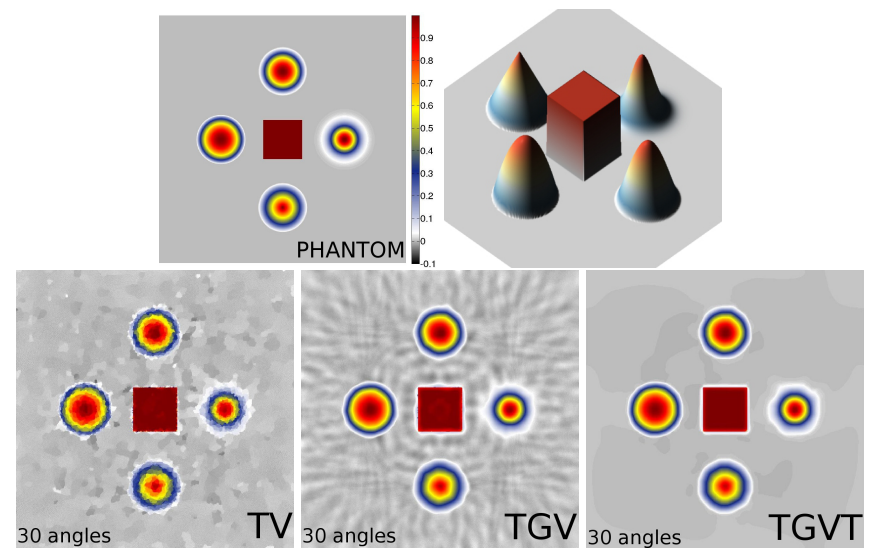

Fig. 1. Top row: phantom No. 1 of $512 \times 512$ pixels size; bottom row: reconstructions from 30 projections with $\mathrm{TV}$, TGV and TGVT methods; Note the significant improvement of the TGVT method in removing background oscillations compare to the TV and TGV reconstructions.

timized parameters we performed 1000 iterations to demonstrate convergence plots of $\Delta_{1}$ with respect to iteration number for the TV, TGV and the TGVT algorithms (see Fig. 2 (left)). It can be seen that the TGVT method has the lowest error for reconstruction from only 30 projection angles (see Table 1 and the convergence speed is also improved. The reconstructed images are shown in Fig. 11(bottom row). Reconstruction with the TV penalty results in a rugged piecewiseconstant appearance, while both TGV and TGVT methods provide desirable piecewise-smooth recovery. Some ringing artifacts are visible in the background of the TGV recovery (influence of the second order gentle penalization) while the
Table 1. NRMSE values for TV, TGV and TGVT methods

\begin{tabular}{|l|l|l|l|}
\hline & TV & TGV & TGVT \\
\hline Phantom 1 (30 proj.) & 0.071 & 0.055 & $\mathbf{0 . 0 3 9}$ \\
\hline Phantom 2 (90 proj.) & 0.156 & 0.153 & $\mathbf{0 . 1 4 2}$ \\
\hline Phantom 2 (75 proj., la) & 0.231 & 0.234 & $\mathbf{0 . 2 1 3}$ \\
\hline
\end{tabular}
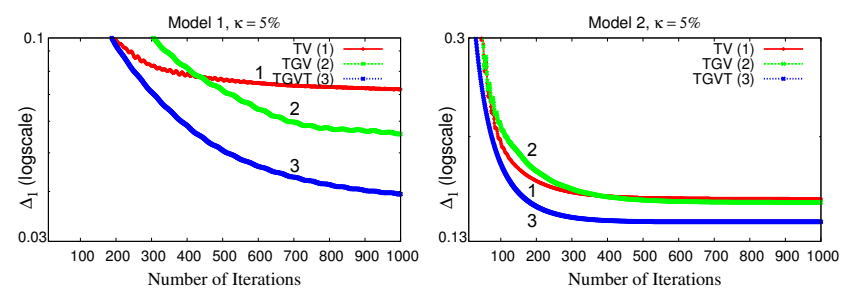

Fig. 2. Convergence plots for the TV, TGV and the TGVT methods; left: model 1; right: model 2.

TGVT method removes them very well (which also results in the substantially lower total error).

The next experiment involves the reconstruction of the more realistic phantom which was constructed from the high resolution scan of the bone-bioglass sample acquired at the DLS Diamond-Manchester Branchline (see Fig. 3 (top row, left)). The original high resolution image was reconstructed,

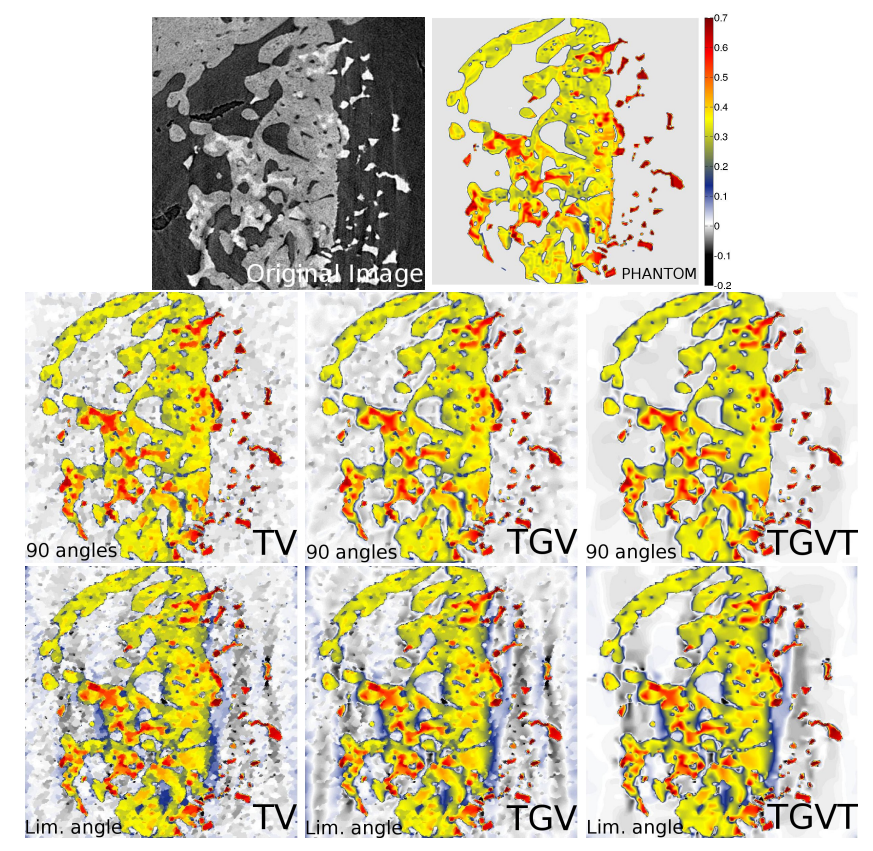

Fig. 3. Top row: The original image and the phantom No. 2 of $512 \times 512$ pixels size; middle row: reconstructions from 90 projections; bottom row: limited angle reconstructions from 75 projections when 30 angles missing in $[0, \pi)$ angular range.

denoised and segmented into three phases (air, bone and bioglass). The bone structure with some pieces of bioglass (high 
absorption material) is then cropped to form phantom No. 2 (see Fig. 3 (top row, right)).

The phantom No. 2 was reconstructed using 90 full projections and 75 limited angle (30 angles missing in $[0, \pi)$ angular range) projections (see Fig. 3. Random noise $(\kappa=5 \%)$ has been added to the projections. The TV reconstructions are again quite blocky and many smooth features, such as the bioglass inclusions, are of "cartoon" appearance. The TGV method provides a much better recovery of smooth features, however some streak artifacts (due to missing wedge) are more emphasized than with the TV method. The TGVT method successfully suppresses background oscillations and reduces streak artifacts. The total error is again less with the proposed method as it can be seen from the Table 1 . Convergence plots for phantom No.2 are presented in Fig. 2 (right). To better appreciate the differences in quality of reconstruction, the magnified images are shown in Fig. 4. The TGVT reconstruction is smooth and almost artifacts free.

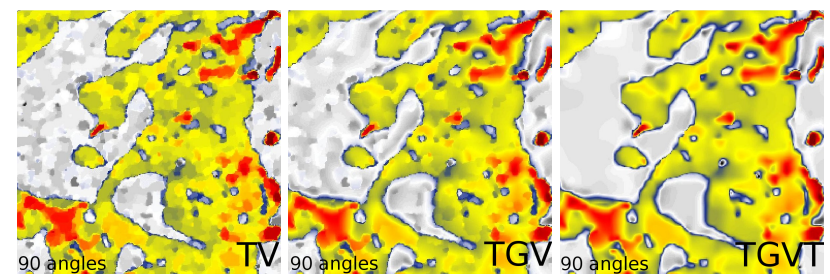

Fig. 4. The magnified images reconstructed with the TV, TGV and the TGVT methods (cropped from the middle row of Fig. 3). The background oscillations are suppressed better with the TGVT method while resolution is maintained and the piecewise-smooth nature is preserved.

\section{DISCUSSION AND CONCLUSIONS}

Since the proposed method is heuristic and does not attached to any specific cost function minimization it is difficult to draw conclusions regarding its convergence properties. Each IHT step is a projection of the problem onto one of several subspaces where the problem is convex, the main difficulty is to show that we do not switch spaces ad infinitum. However, as long as we iterate within the subspace convergence is ensured (provided optimal $\tau$ ). Ultimately, for sparser solutions with the TGVT method we pay the price of non-convexity.

Wavelet recovery is piecewise-smooth and it fits well to our assumptions of the object. However, wavelets might not be the best choice of basis functions to minimize limited angle streak artifacts. Usually those streaks are related to the scanning positions and therefore directional. Curvelets might be a better choice to further suppress these artifacts since they consider various geometrical orientations of a signal. Overall, the method is simple and the use of the fast wavelet transform does not add any substantial computational cost to it.
In this paper we presented a novel reconstruction approach which combines the state-of-the-art TGV penalty and IHR techniques within the CP primal-dual iterative method. The proposed modification is a one-step operation which fits well the CP reconstruction strategy. Our preliminary experiments show that the aliasing artifacts can be reduced and noise influence can be further minimized without any loss of resolution (preserving the piecewise-smooth nature of the recovery). Our future work will be dedicated to better understand the properties of the method and its application to the real datasets.

\section{REFERENCES}

[1] C. R. Vogel, Computational methods for inverse problems, vol. 23, 2002.

[2] L. Rudin, S. Osher, and E. Fatemi, "Nonlinear total variation based noise removal algorithms," Physica D, vol. 60(1-4), pp. 259-268, 1992.

[3] M. N. Wernick and J. N. Aarsvold, Emission tomography: the fundamentals of PET and SPECT, 2004.

[4] R. H. Chan, H. Liang, S. Wei, M. Nikolova, and X. C. Tai, "High-order total variation regularization approach for axially symmetric object tomography from a single radiograph," Inverse Problems and Imaging, vol. 9(1), pp. 55-77, 2015.

[5] K. Bredies, K. Kunisch, and T. Pock, "Total generalized variation," SIAM Journal on Imaging Sciences, vol. 3(3), pp. 492-526, 2010.

[6] T. Blumensath and M. E. Davies, "Iterative hard thresholding for compressed sensing," Applied and Coтputational Harmonic Analysis, vol. 27(3), pp. 265-274, 2009.

[7] A. Chambolle and T. Pock, "A first-order primal-dual algorithm for convex problems with applications to imaging.," Journal of Mathematical Imaging and Vision, vol. 40(1), pp. 120-145, 2011.

[8] E. Y. Sidky, J.H. Jørgensen, and X. Pan, “Convex optimization problem prototyping for image reconstruction in computed tomography with the chambollepock algorithm," Physics in medicine and biology, vol. 57(10), pp. 3065, 2012.

[9] I. W. Selesnick, R. Baraniuk, and N. G. Kingsbury, "The dual-tree complex wavelet transform," Signal Processing Magazine, IEEE, vol. 6, pp. 123-151, 2005.

[10] D. Kazantsev, CP-TV-TGV-TGVT software pack, Available at http://ccpforge.cse.rl.ac. uk/gf/project/ccpi_itr/frs/?action= FrsReleaseView\&release_id=527 